\title{
«Une élection purement populaire » : l'impact de la démocratie sur les élections municipales dans les plus grandes villes à l'automne 1792
}

A Purely Popular Election: the impact of Democracy on the Municipal Elections in the largest towns in the Autumn of 1792.

\section{Melvin Edelstein}

\section{OpenEdition}

Journals

\section{Édition électronique}

URL : https://journals.openedition.org/ahrf/11206

DOI : 10.4000/ahrf.11206

ISSN : 1952-403X

\section{Éditeur :}

Armand Colin, Société des études robespierristes

\section{Édition imprimée}

Date de publication : 1 septembre 2007

Pagination : $29-49$

ISSN : 0003-4436

\section{Référence électronique}

Melvin Edelstein, « «Une élection purement populaire » : l'impact de la démocratie sur les élections municipales dans les plus grandes villes à l'automne 1792 », Annales historiques de la Révolution française [En ligne], 349 | juillet-septembre 2007, mis en ligne le 01 septembre 2010, consulté le 01 juillet 2021. URL : http://journals.openedition.org/ahrf/11206; DOI : https://doi.org/10.4000/ahrf. 11206 


\title{
«UNE ÉLECTION PUREMENT POPULAIRE 》 : L'IMPACT DE LA DÉMOCRATIE SUR LES ÉLECTIONS MUNICIPALES DANS LES PLUS GRANDES VILLES À L'AUTOMNE 1792
}

Melvin EDELSTEIN

\begin{abstract}
L'objet de cet article est d'étudier l'impact de la démocratie sur les élections municipales dans les grandes villes à l'automne 1792. Pour certains historiens, l'établissement du suffrage universel masculin a donné le droit de vote aux classes populaires, aboutissant donc à un brusque renversement des dirigeants municipaux. Nous mettons ces arguments à l'épreuve. Cet article s'insère également dans un débat historiographique. D'après $F$. Furet et P. Gueniffey, les élections de 1792 n'étaient pas du tout démocratiques. La faiblesse du vote permit à une minorité agissante, c'est-à-dire les Jacobins, d'organiser le vote. L'étude des élections municipales met en cause les thèses de Furet et Gueniffey. Ils ont négligé de prendre en compte les sections, qui ont joué un rôle aussi important que les clubs. Bien que le suffrage universel ait fait progresser la démocratisation du personnel municipal, l'impact de la démocratie fut plus limité que certains historiens ne le pensent.
\end{abstract}

Mots-clés : suffrage universel masculin, démocratie, élections municipales, grandes villes.

Le décret du 22 septembre 1792 de la Convention nationale ordonnait le renouvellement des corps administratifs, municipaux et judiciaires'. Les élections municipales se déroulèrent selon le décret du 11 août 1792. La distinction entre citoyens actifs et non actifs fut supprimée. Les conditions d'éligibilité exigées pour être élu furent également supprimées. Lâge de vote fut abaissé de vingt-cinq à vingt et un ans ${ }^{2}$. Le suffrage quasiment universel masculin fut donc instauré. Annonçant les prochaines élections municipales, Joseph-Marie Dorvo, procureur de la commune de Nantes,

(1) Jean-Baptiste DUVERGIER, Collection complète des lois, décrets, ordonnances, règlements et avis $d u$ Conseil d'État, 24 vols, Paris, 1825-1828, V, p. 2-3.

(2) Pour avoir le droit de vote, il suffisait d'être Français, âgé de vingt-un ans, domicilié depuis un an, vivant de son revenu ou du produit de son travail, et de n’être pas en état de domesticité. KL., IV, p. 349-350. 
proclama : "Naguère, une ligne odieuse de démarcation séparait les citoyens riches des citoyens indigents ; les derniers étaient privés du droit de choisir leurs magistrats, et l'édilité pouvait encore ne pas venir d'une élection purement populaire ; mais, grâce aux lumières, il n'en est plus ainsi ${ }^{3}$. En fait, ces élections "purement populaires » selon l'expression de J.-M. Dorvo furent les seules élections municipales tenues au suffrage élargi pendant la décennie révolutionnaire.

Au préalable, il faut définir le mot « démocratie ». Dans cet article, le mot démocratie désigne l'élargissement du nombre de citoyens ayant droit de voter. Il s'agit de la suppression de toute condition fiscale et économique exigée pour avoir le droit de vote et être éligible aux fonctions publiques. Le suffrage s'approche donc autant que possible du suffrage universel masculin. De ce fait, de nouvelles couches sociales devaient accéder au droit de vote et à l'éligibilité. Il pouvait se produire un élargissement de la base sociale des votants et des élus.

Les historiens ont étudié avec intérêt l'impact des élections démocratiques sur la Convention, ils se sont moins interrogés sur le renouvellement des corps administratifs, municipaux et judiciaires à l'automne 1792. Le renouvellement des municipalités offre l'occasion d'étudier l'impact de la démocratie. Le suffrage quasiment universel a-t-il démocratisé, c'est-àdire élargi le recrutement social du personnel municipal ? Les historiens français de gauche, de Jean Jaurès à Georges Lefebvre, le croient, mais ils ne s'accordent pas sur la date. D'après Jaurès, le 31 mai 1793 marque un tournant. Il écrit que « c'est à cette date seulement que les premiers cadres administratifs de la Révolution sont brisés et renouvelés ${ }^{4}$. Cependant, selon Albert Mathiez, " après le 10 août 1792, les nouvelles municipalités s'imprégnèrent d'éléments populaires "s. Lefebvre est, lui, du même avis que Jaurès : « Dans les villes, l'artisanat, le commerce de détail, les arts libéraux accédèrent plus ou moins nombreux au conseil général de la commune $[\ldots]$; en 1793 , ils prédominèrent $»^{6}$. La question de la période où se fit cette rupture de la composition sociale des municipalités, est importante pour comprendre la contribution de la Révolution à la démocratie. Si la démocratisation du recrutement social des élus s'est produite en l'an II, ce serait le résultat des mesures prises par les représentants du peuple en

(3) AM Nantes 1 D 6 (Proclamation concentant le renouvellement du Conseil général de la commune, le 26 novembre 1792). Camille MELlinet, La Commune et la Milice de Nantes, 12 vols., Nantes, 1840-1843, VII, p. 49.

(4) Jean JauRÈs, Histoire socialiste de la Révolution française, édition préparée par Albert Mathiez, 7 vols., Paris, 1922-1923, II, p. 64.

(5) Albert Mathiez, La Révolution française, Paris, réimpression, 1989, p. 110. La première édition est parue en 1922.

(6) Georges LefEBVRE, La Révolution française, Paris, 1968, p. 574. 
mission. En revanche, si elle est arrivée à l'automne 1792, ce serait dû aux élections au suffrage élargi.

Pour certains historiens, cet élargissement du suffrage a donné le droit de vote aux classes populaires, c'est-à-dire aux artisans, boutiquiers, marchands détaillants, compagnons et manouvriers, aboutissant donc à un brusque renversement des dirigeants municipaux. Paul Hanson affirme : "L'instauration du suffrage universel masculin aux élections de l'automne 1792 renversa la composition des conseils municipaux partout en France, nulle part plus dramatiquement qu'à Lyon et à Marseille »?. Si ces élections marquèrent en même temps une radicalisation politique et un bouleversement de la composition sociale des conseils municipaux dans beaucoup de villes, c'est une situation opposée qui se produisit à Paris. Le nouveau conseil général fut plus modéré que le précédent ${ }^{\kappa}$. Nous allons mettre ces arguments à l'épreuve.

Étudions donc les élections municipales dans les grandes villes, élections qui ont commencé en octobre 1792 et pris fin en août 1793 dans le cas particulier de Paris', pour mesurer l'impact du processus de démocratisation sur leurs résultats. À l'opposé des renouvellements partiels des années précédentes, ces scrutins ne se déroulèrent pas simultanément. De plus, ils n'ont pas suivi les mêmes procédures". En prenant appui sur les premières élections municipales dans les dix plus grandes villes en 1790, nous pouvons faire des comparaisons avec le début de la Révolution et également avec les élections en 1791 et l'année suivante". Nous nous poserons les questions suivantes : 1) Le nombre de votants a-t-il augmenté ? Les classes populaires urbaines ont-elles pris un plus grand intérêt à ces élections ? 2) Y a-t-il eu un renouvellement complet du personnel municipal ? Quel pourcentage de l'ensemble des élus représentaient les nouveaux élus, n'ayant jamais accédé à un poste municipal ? 3) Y a-t-il eu démocratisation du recrutement social des membres du conseil général ? Jusqu'à quel point les artisans et les boutiquiers étaient-ils majoritaires dans le conseil municipal ? 4) Y avait-il une radicalisation politique des édiles ?

(7) Paul HAnson, The Jacobin Republic Under Fire: The Federalist Revolt in the French Revolution, University Park, Pennsylvania, 2003, p. 11.

(8) Trés peu des commissaires de la Commune du 10 Août furent reconduits. Idem., p. 42.

(9) Les élections municipales ont commencé le 5 octobre à Paris, le 23 octobre à Toulouse, le 5 novembre à Lyon, en novembre à Lille, en décembre à Nantes, fe 16 décembre à Versailles, le 23 décembre à Rouen et le 30 décembre à Marscille.

(10) Le décret du 14 déccmbre 1792 ayant supprimé le troisième tour, s'il y avait ballotage, c'était au second tour.

(11) Melvin EdELsteIN, " "Laying the Foundations for the Regeneration of the Empire" : The first Municipal Elections in the Biggest Citics of France during the Revolution ", French History, 17 , Scptember 2003 , p. $251-279$. Versailles, qui fut au dixième rang en 1790 ne fut qu'au quatorzième rang en 1794. Pour la population des villes en 1794, voir Bernard LEPETIT, Les villes dans la France moderne (1740-1840), Paris, 1988, p. 450-453. 
Nous nous intéressons à l'impact de la démocratie instaurée en 1792 sur les élections municipales, mais nous nous intéresserons également à un débat historiographique. D'après François Furet et Patrice Gueniffey, les élections de 1792 n'ont pas du tout été démocratiques ${ }^{12}$. Suivant le modèle d'Augustin Cochin, ils affirment que la faiblesse du vote permit à une minorité agissante, c'est-à-dire les Jacobins, d'organiser ces élections. Elles n'étaient nullement libres, la liberté de choix des votants étant entravée. Cela revient à dire que ces élections furent captées et manipulées. Ces auteurs négligèrent pourtant les élections locales. Létude des élections municipales conforte-elle leurs thèses ?

Compte tenu du système du vote direct dans les sections de la ville même, la vive politisation, l'intégration des citoyens antérieurement non actifs, nous pourrions attendre une forte mobilisation, comme le pense Hanson. Il faut toutefois rappeler, comme nous l'avons démontré, que les historiens ont surestimé l'élargissement du corps électoral ${ }^{13}$. Les listes des citoyens actifs étant périmées, celles de citoyens ayant le droit de vote étant absentes, il n'est pas facile de calculer un taux de participation électorale en 1792. Nous comparerons donc le chiffre le plus élevé de votants pour l'élection du maire en 1792 avec ceux des années précédentes. Ce scrutin a attiré généralement plus de votants. Seuls les chiffres de Lille manquent. À l'exception de Strasbourg, le nombre de votants a très largement dépassé le chiffre atteint en novembre 1791. À Toulouse, les votants sont passés de 398 à 2007 . Ils ont doublé à Rouen et Versailles et approximativement triplé à Bordeaux. Cette forte augmentation s'explique peut-être par le fait que, parfois, les votants ont réellement eu le choix entre deux candidats opposés ${ }^{14}$. Les élections au suffrage élargi auraient, semble-t-il, mobilisé les votants.

(12) François Furet, Penser la Révolution française, Paris, 1983. François FuRET et Denis Richet, La Révolution française, Paris, 1965. Patrice GUENIFFEY, Le nombre et la Raison : La Revolution française et les élections, Paris, EHESS, 1993.

(13) Melvin EdelsTEıN, " Létablissement de la République en Côte-d'Or : étude électorale et politique " dans Révolution et République L'exception française, sous la direction de Michel Vovfiı... Paris, 1994, p. 226-227. Serge AвERDAM, « Lélargissement du droit de vote entre 1792 et 1795 au travers du dénombrement du Comité de division et des votes populaires sur les constitutions de 1793 et 1795 ", thèse pour le doctorat, Université de Paris I, 2001. Serge ABERDAM, Démographes et démocrates. L'ceuvre du comité de division de la Convention nationale, Paris, SER, 2004.

(14) C'est le cas à Lyon. À l'issue du premier tour qui a attiré 5787 votants, Joseph Chalier, jacobin radical, fut en tête. Effrayés, les modérés sont apparus au second tour : 9012 votants se sont mobilisés. Nivèvre-Chol, rolandin, a largement battu Chalier. Ville de Lyon. Procès-verbaux des séances des corps municipaux de la ville de Lyon publiés par la municipalité, d'après les manuscrits originaux 1787-an VIII, 8 vols., Lyon, 1900-1904, III, p. 353-391. W.D. Edmonds, Jacobinism and the Revolt of Lyon, 1789-1793, Oxford, 1990, p. 138-139. 
Tableau 1 : Participation électorale aux scrutins pour le maire : 1790, 1791 et 1792

\begin{tabular}{|c|c|c|c|}
\hline VLLE & 1790 & 1791 & 1792 \\
\hline 1. BORDEAUX & 3737 & 1292 & 3497 \\
\hline 2. LILLE & 1783 & $\mathrm{~N} / \mathrm{D}$ & $\mathrm{N} / \mathrm{D}$ \\
\hline 3. LYON & 5953 & 3573 & 9012 \\
\hline 4. MARSEILLE & 4409 & 1928 & 4271 \\
\hline 5. NANTES & 1510 & 701 & 876 \\
\hline 6. PARIS & 14010 & 10632 & $\begin{array}{r}\text { (15 474) dans } \\
47 \text { sections; } \\
15191 \text { dans } \\
48 \text { sections en } \\
\text { février } 1793\end{array}$ \\
\hline 7. ROUEN & 2529 & 867 & 1747 \\
\hline 8. STRASBOURG & 5685 & 4993 & 2508 \\
\hline 9. TOULOUSE & 1732 & 398 & 2007 \\
\hline 10. VERSAILLES & 1918 & 1169 & 2135 \\
\hline
\end{tabular}

La comparaison du nombre de participants de 1792 avec celui de 1790 nous autorise à en douter. Il n'est supérieur que dans quatre villes ; il est inférieur dans quatre villes. La situation à Marseille est ambiguë ${ }^{15}$. À première vue, l'élargissement du suffrage paraît avoir mobilisé, mais il faut admettre que le nombre de votants n'est qu'une part infime des votants potentiels ${ }^{16}$.

(15) Le vote est supérieur dans les quatre villes suivantes : Lyon, Paris, Toulouse et Versailles. Il est inféricur dans les quatres villes suivantes: Bordeaux, Nantes, Rouen et Strasbourg. Le nombre de votants marseillais $(4271$ ) est inférieur à cclui de 1790 (4 409), mais en 1792, le vote n'est enregistré que pour 31 des 32 sections. Il parait possible donc d'inclure Marseille dans le premier groupe. Pour les votes en 1792. voir AM Bordeaux K 4 et Alan ForResr, Society and Politics in Revolutionary Burdeaux, Oxford, 1975, p. 40); Ville de Iyon. Procès-verhaux des séances des corps municipaux de la ville de Lyon, op. cit., III, p 353391; AM Marseille 3 K 8 et I D 10-11; MelLINET, La Commune et la milice de Nantes, op. cit., VII, p. 47-48; BM Rouen K 5-6 et Ville de Rouen. Analyses des délibérations de l'Assemblée municipale et électorale du 16 juiller au 4 mars 1790 et du conseil général de la commune du 4 mars 1790) au 25 brumaire an IV (6 novembre 1795), Rouen, 1905, p. 89-96; AM Strasbourg 204 MW 52; AM Toulouse I K 11 ; AM Versailles 1 K 1286. Pour le chiffre de 15474 votants de 47 des 48 sections parisiennes, voir P.J.B. BuCHEz et P.C. Roux, Histoire parlementaire de la Révolution française, 40 vols., Paris, 1834-1838, XX, p. 59.

(16) On estime que le nombre de 15474 votants parisiens dans 47 des 48 sections, chiffre le plus élevé atteint jusqu'en 1793, représente moins de dix pour cent des votants potentiels. Pour les 15474 votes pour le maire dans 47 des 48 sections, voir Réimpression de l'ancien Moniteur Universel, XIV, N" 291, 17 octobre 1792. D'aprés MorTimer-TounnalJX : " des 160000 électeurs ayant droit de vote, 14137 seulement, c'est-à-dire moins du dixiensc prisent part au scrutin du 4 octobre [1792] ». Louis Mortimer-Tournaux, Histoire de la Terreur 1792-1794 d'après les documents inédits, 8 vols., Paris, 1868-1881, V, p. 95. 
Létude de la composition sociale des conseils généraux des villes concernées doit s'organiser en deux parties : le corps municipal et les notables, de moindre importance. À la tête de la municipalité, on trouve le maire, le procureur de la commune et son substitut, dont deux furent élus à Paris. Cet échelon est marqué par la continuitér. Deux maires seulement sur dix ont été de nouveaux élus. Ils étaient toutefois des hommes expérimentés ${ }^{18}$. La composition sociale n'en a été aucunement ébranlée ${ }^{19}$. Aucun artisan ou boutiquier ne fut élu. Sur le plan politique, ces élus étaient généralement modérés. Certes, six maires étaient jacobins, mais quatre n'étaient pas sociétaires ${ }^{20}$. Quant aux procureurs de ces communes, on ne perçoit que peu de changement. Trois seulement étaient de nouveaux élus $^{21}$. Huit étaient des hommes de loi ; deux étaient journalistes. Sur le plan politique, on dénombre quatre jacobins, un cordelier et un militant du Club central lyonnais ${ }^{22}$. Parmi les substituts quatre seulement étaient de nouveaux élus, mais nous manquons de renseignements sur deux d'entre eux. La composition sociale fut effectivement modifiée. Seuls trois hommes de loi furent élus. Il y eut toutefois trois membres des professions intellectuelles, un bourgeois et un seul artisan ${ }^{23}$. Sur le plan politique, on trouve des militants tels que Hébert à Paris et Berthelon à Lyon et au moins quatre jacobins. Leétude des dirigeants révèle combien l'impact du suffrage élargi fut limité. La continuité est nette ; il y a très peu de nouveaux élus. La composition sociale n'est guère modifiée. Si le poids de la basoche est moindre, c'est au profit des professions intellectuelles. Le seul artisan se trouve à l'échelon du substitut. Même si les clubistes sont dominants, il faut noter que nombre d'entre eux se révèlent modérés. La faible démocratisation du recrutement social à la tête de la commune infirme les arguments d'Hanson.

(17) Cinq des maires sortants furent réélus, mais trois d'entre eux ont démissionné. Six maires étaient d'anciens officiers municipaux ; deux seulement étaient de nouveaux élus.

(18) Baco, le maire nantais, ćtait un ancien député du Tiers ćtat; Rondeaux, maire rouennais, était membre du directoire du département.

(19) Il y avait trois hommes d'affaires, trois hommes de loi, deux membres des professions intellectuelles, scientifiques et artistiques, un médecin et un rentier.

(20) Parmi les clubistes, Baco a adhéré au club Feuillant, Nivèvre-Chol fut rolandin, Mourraille fut arrêté à cause des dénonciations des jacobins et des sections marseillaises et Derry fut guillotiné comme meneur fédéraliste. Parmi les non-sociétaires, Saige fut guillotiné comme meneur fédéraliste, tandis que Turckheim, fils fut suspendu le 18 janvier 1793 .

(21) Six des sortants furent réélus, un était ancien subsitut du procureur de la commune, trois seulement étaient de nouveaux élus.

(22) Un autre fut membre de la société scissionnaire du cluh jacobin strashourgeois. Trois n'étaient pas clubistes.

(23) Nous manquons de renseignements sur deux d'entre cux. Quant à la composition sociale, quatre avaient des professions indéterminées. 
Tableau 2 : Les maires des dix plus grandes villes (de 1790) élus en 1792

\begin{tabular}{|c|c|c|c|}
\hline VILLE & NOM & PROFESSION & $\begin{array}{l}\text { CARRIERE } \\
\text { POLITIQUE }\end{array}$ \\
\hline 1. BORDEAUX & $\begin{array}{l}\text { SAIGE, } \\
\text { François-Armand }\end{array}$ & $\begin{array}{l}\text { Fils d'un secrétaire } \\
\text { du roi, avocat-général } \\
\text { au Parlement, mais } \\
\text { depuis } 1778, \text { négociant }\end{array}$ & $\begin{array}{l}\text { Réélu ; non jacobin; } \\
\text { exécuté le } 25 \\
\text { octobre } 1793\end{array}$ \\
\hline 2. LILLE & $\begin{array}{l}\text { LEFEBVRE- } \\
\text { DHENIN, } \\
\text { Guillaume-Joseph }\end{array}$ & Rentier & $\begin{array}{l}\text { Officier municipal ; } \\
\text { non-jacobin }\end{array}$ \\
\hline 3. LYON & $\begin{array}{l}\text { NIVIERE-CHOL, } \\
\text { Antoine }\end{array}$ & Négociant & $\begin{array}{l}\text { Officier municipal } \\
\text { élu maire, il donna } \\
\text { sa démission le } 7 \\
\text { février } 1793 \text {, mais } \\
\text { réélu, il refusa ; } \\
\text { jacobin (brissotin) }\end{array}$ \\
\hline 4. MARSEILLE & \begin{tabular}{|l|} 
MOURRAILLE, \\
Jean-Raymond-Pierre
\end{tabular} & $\begin{array}{l}\text { Astronome, } \\
\text { académicien }\end{array}$ & $\begin{array}{l}\text { Réélu ; président du } \\
\text { club jacobin, } \\
\text { dénoncé par les } \\
\text { sections et les } \\
\text { jacobins, puis arrêté } \\
\text { le } 12 \text { avril } 1793 \text {, mais } \\
\text { acquitté par le } \\
\text { Tribunal populaire }\end{array}$ \\
\hline 5. NANTES & $\begin{array}{l}\text { BACO DE } \\
\text { LA CHAPELLE, } \\
\text { René-Gaston }\end{array}$ & $\begin{array}{l}\text { Avocat au Parlement } \\
\text { et procureur du roi } \\
\text { au présidial }\end{array}$ & $\begin{array}{l}\text { Député à } \\
\text { l'Assemblée } \\
\text { constituante, } \\
\text { jacobin, il passa } \\
\text { aux Feuillants }\end{array}$ \\
\hline 6. PARIS & $\begin{array}{l}\text { CHAMBON, } \\
\text { Nicolas }\end{array}$ & Médecin & $\begin{array}{l}\text { Notable, puis officier } \\
\text { municipal; jacobin } \\
\text { en } 1790 ; \\
\text { démissionnaire } \\
\text { le } 2 \text { février } 1793 \text {; } \\
\text { remplacé par } \\
\text { Jean-Nicolas } \\
\text { PACHE, } \\
\text { Ministre de } \\
\text { la Guerre }\end{array}$ \\
\hline
\end{tabular}




\begin{tabular}{|c|l|l|l|}
\hline VILLE & \multicolumn{1}{|c|}{ NOM } & \multicolumn{1}{|c|}{ PROFESSION } & \multicolumn{1}{c|}{$\begin{array}{c}\text { CARRIERE } \\
\text { POLITIQUE }\end{array}$} \\
\hline 7. ROUEN & $\begin{array}{l}\text { RONDEAUX, } \\
\text { Charles-Marie- } \\
\text { François }\end{array}$ & $\begin{array}{l}\text { Sieur de Montbray, } \\
\text { seigneur de Brilly, } \\
\text { Conseiller du roi, } \\
\text { maître ordinaire de la } \\
\text { chambre des comptes }\end{array}$ & $\begin{array}{l}\text { Administrateur } \\
\text { au directoire du } \\
\text { département } \\
\text { jacobin en 1790, } \\
\text { il fut arrêté le 19 } \\
\text { octobre 1793, } \\
\text { mais il fut libéré le } \\
\text { 7 fructidor an II }\end{array}$ \\
\hline 8.STRASBOURG & TURCKHEIM, fils, \\
Bernard-Frédéric & Banquier & $\begin{array}{l}\text { Officier municipal, } \\
\text { destitué comme } \\
\text { officier municipal le } \\
20 \text { août 1792, } \\
\text { non-jacobin; destitué } \\
\text { comme maire le 18 } \\
\text { janvier 1793 }\end{array}$ \\
\hline 9. TOULOUSE & $\begin{array}{l}\text { DERREY DE } \\
\text { BELBEZE, Marc }\end{array}$ & Conseiller au sénéchal & $\begin{array}{l}\text { Officier municipal, } \\
\text { guillotiné le 29 juin } \\
1794 ; \text { ancien } \\
\text { président du club } \\
\text { jacobin. }\end{array}$ \\
\hline 10. VERSAILLES & HUVE, Jean-Jacques & Architecte ; inspecteur \\
des bâtiments du Roi & $\begin{array}{l}\text { Officier municipal, } \\
\text { destitué le 30 } \\
\text { septembre 1793, } \\
\text { non-jacobin }\end{array}$ \\
\hline
\end{tabular}

Lavènement du suffrage élargi a-t-il bouleversé la composition des officiers municipaux, comme Hanson le pense ? Notre analyse concerne neuf villes : Paris sera étudiée séparément. Le renouvellement est important : $44 \%$ étaient des nouveaux élus. Cependant, la moyenne masque des variation $\mathrm{s}^{24}$. Ce changement du personnel issu du suffrage élargi est pourtant moins radical que celui produit en novembre 1791, lorsque les nouveaux élus représentaient $68 \%$. Bien que le changement du personnel soit moindre que ce que l'on pense, la démocratisation du recrutement social est réelle. Admettons d'emblée que tout classement social comporte une part d'arbitraire, la catégorisation professionnelle s'avérant difficile.

(24) $28 \%$ seulement étaient réélus et $28 \%$ étaient des notables. Cependant, la moyenne masque des variations considérables : plus de la moitić ćtaient des nouveaux élus à Lyon, Marseille et Rouen, mais il y en avait très peu à Bordeaux, Toulouse et Versailles.

(25) Deux pour cent seulement étaient réélus et $30 \%$ étaient des notables. 
Le problème le plus épineux est la frontière entre ce que j'appelle les " hommes d'affaires » et les artisans, boutiquiers et marchands détaillants. La méthode utilisée ici est la même que celle de notre article précédemment citée ${ }^{2 t}$. Les privilégiés ont presque disparu. Si les hommes d'affaires sont toujours au premier rang, leur représentation a baissé de $45 \%$ en 1790 à $38 \% \%^{27}$. Les artisans et boutiquiers sont passés du troisième rang en 1790 au second rang. La thèse d'Hanson est confortée par l'augmentation de leur représentation : $8 \%$ en 1790 à un quart ${ }^{2 \aleph}$. La basoche a chuté du second rang avec un quart en 1790 à $7 \%{ }^{24}$. A l'échelon des officiers municipaux, la démocratisation du recrutement social a progressé, mais pas uniformément. Si les artisans et boutiquiers sont entrés dans le corps municipal, ils n'étaient majoritaires qu'à Lyon et peut-être aussi à Marseille.

C'est à l'échelon des notables que la démocratisation du recrutement social a fait le plus de progrès. Le renouvellement est très important : $63 \%$ sont de nouveaux élus ${ }^{31}$. Ce bouleversement du personnel est toutefois moins spectaculaire que le raz de marée qui eut lieu en novembre 1791 : $90 \%$ étaient alors de nouveaux élus ${ }^{31}$. Linstauration du suffrage élargi a bouleversé la composition du conseil général. Les artisans et boutiquiers représentent désormais l'élément le plus important, alors qu'ils étaient au second rang. Ils ont progressé de $18 \%$ en 1790 à $35 \%$ en $1792^{32}$. Les hommes d'affaires suivent de près avec $30 \%$, alors qu'ils étaient pourtant au premier rang les années précédentes avec $34-35 \%$. La basoche a chuté

(26) Melvin EDELSTEIN, « Laying the Foundations ", op. cit., p. 260 et p. 278-279.

(27) Il n'y a qu'un ministre protestant et un ci-devant marquis. Parmi les hommes d'affaires, il y a : cinquante négociants, trois fabricants, deux marchands, deux marchands de draps (Versailles), un courtier, un ancien capitaine (Marseille), un marchand de dentelles (Lille) el un marchand mercier (Versailles). Les hommes d'affaires sont toujours prédominants dans quatre villes: Bordeaux, Lille, Rouen et Nantes.

(28) Ils étaient déjả $14 \%$ en 1791-1792. Parmi les artisans et boutiquicrs il y a trois imprimeurs, trois fabricants, ouvriers en soie (Lyon), deux marchands épiciers, dcux faiseurs de bas (Lyon), deux peseurs, deux entrepreneurs, deux épiciers, un constructeur, un pâtissier, un vitrier, un chapelier, un gazier, un vinaigrier, un revendeur de meubles, un auffier, un bonnetier, un horloger, un teinturier, un droguiste, un marchand tapissicr, un vitrier, un serrurier, un marchand chapelier, un marchand chaussetier, un menuisier. un ferblantier, un cabaretier, un peigneur, un marchand de vin, un boulanger, un graveur et un marchand farinicr. Les artisans et boutiquiers représentent plus que la moitić à Lyon et $35 \%$ au minimum à Marseille (pour quatre ćlus, la profession est indéterminée). En revanche, très peu d'artisans ont été élus dans six villes : Bordeaux, Lille, Nantes, Rouen, Strasbourg et Toulouse.

(29) La basoche n’était que $18 \%$ en 1791 . Le fait que les représentants des professions intellectuelles et les médecins soient plus nombreux que les hommes de loi conforte les arguments de Lynn HuNT. Voir Lynn HUNT, Politics, Culture, und Class in the French Revolution, Berkeley, 1984, p. 165. La proportion des hommes de loi, des professions intellectuelles et de la médecine a baissé d'un peu plus d'un tiers les années précédentes à un cinquième.

(30) Un quart est réćlu et $12 \%$ sont des officiers municipaux ou autres.

(31) Nos chiffres s'appliquent à neuf villes; les notables de Lille manquent. $4 \%$ seulement étaient rélus et $6 \%$ étaient officiers municipaux.

(32) Ils ont progressé déjà à $28 \%$ en 1791 . Cette démocratisation ne fut pas uniforme. Majoritaire en trois ou quatre villes : Lyon, Marseillc, Strasbourg ct peut-être Versailles où la profession de onze membres du conseil est indéterminéc, leur représentation est infime ou inférieure à la moyenne dans cinq villes : Bordeaux, Lille, Nantes, Rouen et Toulouse. 
de $21 \%$ en 1790 à $8 \%$ seulement ${ }^{33}$. Presque absents à la tête de la commune, les artisans et boutiquiers sont un quart des officiers municipaux, mais $35 \%$ de notables. La composition socio-professionnelle du conseil général de la commune a été démocratisée, comme le pense Hanson, mais le changement ne fut pas aussi profond qu'il l'indique.

Nous allons étudier plus particulièrement les trois villes où la radicalisation politique et le bouleversement social furent le plus poussés et puis quatre villes où ce fut le contraire. Pour les premières, il s'agit de Paris, Lyon et Marseille ; les quatre dernières sont Strasbourg, Bordeaux, Rouen et Toulouse. Lille, Nantes et Versailles ressemblent à ces dernières villes. Nulle part la démocratisation ne fut plus dramatique qu'à Lyon et Marseille, affirme Hanson. Dans ces deux villes, les jacobins proposèrent des listes de candidats et réussirent à mobiliser les votants, pour une large part les artisans et les ouvriers ${ }^{34}$. Le fait de classer Paris avec Lyon et Marseille peut paraître contradictoire puisque, selon Hanson, Paris s'opposait à la radicalisation générale des conseils municipaux.

Comment Paris pouvait-elle être considérée comme modérée ? Se basant sur Gueniffey, Hanson considère que les résultats électoraux des 28 et 29 novembre 1792 sont le rejet des hommes du 10 août $t^{35}$. Il ne se base que sur les élections de la Commune provisoire, en omettant les scrutins suivants. Lélection du maire confirme son propos, mais son interprétation des résultats électoraux de la Commune provisoire est contestable quand on observe les élections du procureur, des substituts et de la Commune définitive. Pour élire le maire, il a fallu sept scrutins, échelonnés du 5 octobre au 30 novembre. Pétion, le maire démissionnaire, fut réélu, mais il opta pour la Convention ${ }^{30}$. Enfin, Chambon, candidat de Brissot, battit Lulier, le candidat jacobin ${ }^{37}$. Le 11 février 1793, il fallut donner un successeur à Chambon, démissionnaire. La victoire triomphale de Pache, naguère ministre de la Guerre écarté par les Girondins, fut un échec pour les modérés ${ }^{3 *}$.

(33) Ils furent $29 \%$ en 1790 et $19 \%$ en 1791 . Les hommes de loi, les professions intellectuelles et les médecins de $16 \%$ en 1791 et $29 \%$ en $17 \%$ sont tombés à $14 \%$.

(34) Hanson, The Jacobin Republic Under Fire, op. cit., p. 237.

(35) Selon Gueniffey, de 288 sortants, seuls 45 étaient réélus. Patrice Guennifey, " La Communc de Paris " dans Dictionnaire critique de la Révolution française, sous la direction de François Furet et Mona Ozouf, Paris, 1988, p. 522. D'après Hanson, "The new council was more moderate than its predecessor, with fewer than 20 percent of the incumbents reelected to office ". Hanson, The Jacobin Republic Under Fire, op. cit., p. 42.

(36) Comme maire, il fut réduit à l'effacement. Rejeté par les électeurs du département de Paris, ce girondin fut élu député du département de l'Eure-et-Loir.

(37) Sigismond LAcrolX, "Lélection du maire de Paris en 1792 ", Révolution française, XXXVIII, 1900, p. 500-522; Atlas de la Révolution française, Tome 11, Paris, sous la direction d'Émile DucoudraY, Raymonde MONNIER et Daniel Roche, Paris, 2000, p. 55.

(38) Réimpression de I'Ancien Moniteur Universel, XV 47 (16 février 1793). Au premier tour, les 48 sections ont fourni 15191 votants, sur lesquels Pache obtenait 11881 voix. 
À l'opposé de Gueniffey et Hanson, trois historiens ne constatent pas dans ces élections un rejet des hommes du 10 Août. D'après Frédéric Braesch et Michel Eude, ces élections furent "franchement montagnardes ». Selon Maurice Genty : "le nouveau conseil était dominé par les démocrates tout autant, sinon plus, que la Commune insurrectionnelle $»^{39}$. Le parquet fut « encore plus montagnard »: Chaumette fut élu procureur, avec, pour substituts, Réal et Hébert. La Commune provisoire installée, les élections de la Commune définitive pouvaient avoir lieu. Plus de sept mois devaient cependant s'écouler avant qu'une nouvelle municipalité puisse s'installer. Ce n'est que le 13 juillet, après la chute de la Gironde, que la liste des cent quarante-quatre notables fut imprimée. D'après Paul SainteClaire Deville : " la Commune est du parti vainqucur, celui des Montagnards, qu'elle a puissamment aidé ${ }^{40}$. Le personnel municipal était renouvelé et composé pour un peu plus de la moitié de nouveaux élus. Trente-neuf seulement avaient fait partie de la Commune provisoire. Les hommes du 10 Août sont revenus en nombre. Ces élections avaient abouti à l'élimination de cent quatre membres de la Commune provisoire. Les hommes du 10 Août revenaient plus nombreux à la maison commune. Vingt-sept d'entre eux, s'ajoutant aux vingt-quatre précédemment mentionnés, formaient un bloc de cinquante-et-un hommes du 10 Août. Une dernière catégorie comprenait les gens qui n'avaient appartenu, ni à la Commune du 10 Août, ni à la Commune provisoire. Soixante-dix-sept élus pourraient être qualifiés de nouveaux élus ${ }^{41}$. À ce changement profond

(39) Frédéric Braesch, Commune du Dix Aout 1792. Etude sur l'histoire de Paris du 20 Juin au 2 décembre 1792, Paris, 1911, p. 1162, “ On ne peut donc pas constater à ce moment dans les sections de Paris un mouvement hostile à l'ancien personnel de la Commune du 10 août. On peut dire, au contraire, étant donné le nombre relativement considérable des anciens membres de cette Commune qui rentrèrent par élection dans la Commune nouvelle, étant donné surtout le nombre des sections qui les nommèrent, que les élections municipales des 28 et 29 novembre furent franchement montagnardes ». Michel EudE, "La commune robespierriste ", AHRF, X, 1933, p. 414. Voir Maurice GENTY, Paris, 1789-1795. L'apprentissage de la citoyenneté, Paris, 1987, p. 195. "Mais le nouveau conseil était dominé par les démocrates tout autant, sinon plus, que la Communc insurrectionnelle ". Selon BraEsCH, quarante-sept à cinquante et un des cent vingt-deux nouveaux élus étaient membres de la Commune insurrectionnelle. Ils font donc $39-42 \%$ des élus. Se basant sur les chiffres fourni par GUENIFFEY (45 réélus des 288 sortants), Hanson calculait $20 \%$. Comme BRAESCH, EUDF et GENTY calculaient les reconduits dans le pourcentage des 122 élus (39-42\%), alors que GUENIFFEY et HANSON calculaient les élus dans le pourcentage des sortants (20\%), ils arrivent à des conclusions opposées.

(40) Paul Sainte-Claire Deville, La Commune de l'an II, Paris, 1946, p. 26-27. 
dans le personnel correspond une modification très sensible de la composition des notables. Les hommes de loi et les hommes de lettres sont passés de trente-sept dans la Commune provisoire à vingt-et-un. Les prêtres tombèrent de huit à deux. En revanche, d'après Sainte-Claire Deville : « les artisans et des ouvriers » sont trente-deux, alors qu'ils ne dépassaient pas la douzaine dans la Commune provisoire. Il les associe à un autre groupe, celui des trente-deux marchands et négociants. Il y a également quatorze « artistes ${ }^{42}$. Selon M. Genty : "La part des professions intellectuelles s'était réduite à une quarantaine au profit des professions directement liées à l'économie, plus de soixante-dix, soit à peu près la moitié $»^{43}$. Lélection des notables achevée, les sections furent encore convoquées afin de choisir les quarante-huit officiers municipaux ${ }^{44}$. Radicalisée et démocratisée par des luttes acharnées, la municipalité définitive fut finalement installée le 7 août 1793.

Les élections lyonnaises virent la victoire écrasante des radicaux. Leur succès était dû au chômage grandissant des ouvriers en soie et à la crise de subsistances. Seul le poste de maire leur a échappé : Joseph Chalier fut battu par le brissotin Antoine Nivière-Chol. Finalement, les jacobins eurent gain de cause le 9 mars 1793 par l'élection de Bertrand, l'ami de Chalier, mais cette victoire ne fut possible que grâce à l'arbitraire. Le premier vote pour le maire n'avait attiré que 5787 votants. Chalier obtint 2691 suffrages, contre 2041 à Nièvre-Chol. Au second tour, 9012 citoyens votèrent. Nièvre-Chol battit Chalier par 5129 suffrages contre 3 883. Ce dernier donna sa démission le 9 février 1793, pour être à

(41) Idem., p. 98-99. Mes chiffres diffèrent un peu de ceux de Sainte-Claire Deville. Je ne trouve que 39 membres de la Commune provisuire. Quant aux hommes du 10 Août, je n'en trouve que quarante-deux. Il y avait également huit membres d'une municipalité constitutionnelle précédente, dont deux nouveaux élus seulement. Au total, je trouve soixante-dix-huit nouveaux élus. Pour les 144 notables, voir BNF Lhti 1322, Municipalité de Paris. Liste générale des 144 citoyens, élus et agréés par les quarante-huit sections, pour former le Conseil général de la commune (13 juillet 1793). Pour les membres de la Commune provisoire en décembre 1792, voir Almanach national, année commune 1793, I'an IT de la République. Pour les membres de la Commune du 10 août 1792, voir BNF Lb ${ }^{\text {t1 }}$ 1301, Commune de Paris. Tableau général des commissaires des 48 secrions qui ont composé le conseil-général de la commune du dix aoút mil sept cent quatre-vingt-douze, l'an premier de la République française ; $\mathrm{BNF} \mathrm{Lb}{ }^{\text {w1 }} 1295$. Liste de tous les membres du conseil-général de la commune, qui ont été chargés de commissions portant comptabilité ou responsahilité ; BNF Lb" 202, Commune de Paris. Second rappont sur l'emploi des 100000 livres décrétées le vingt-deux Août 1792, pour dépenses extraordinaires de la Commune. Pour le personncl de la Commune du 10 août, voir également la Liste par ordre alphabétique des individus ayant fait partie du conseil général de la commune du 9 aout, à minuit, au 17 août soir dans BraEscil, Commune du dix août 1792, op. cit., p. 245-264. Voir également la Liste alphabétique des membres du Conseil général de la Commune de l'an II à l'époque du 9 thermidor avec l'indication du son de chacun d'eux dans SAINTE-ClaIRE. DevilLE, La Commune de l'an II. op. cit., p. 361-379. Pour les membres de la commune au mois de ventóse an II (février-mars 1794), voir EUDE, " La commune robespierriste, »AHRF, XI, 1934, p. 323-347.

(42) Paul Sainte-Claire Deville, La Commune de l'an H, op. cil., p. 99-102.

(43) Maurice GeNTY, Paris, 1789-1795, op. cil., p. 200.

(44) Aucune liste des quarante-huit officiers municipaux n'existe. Cependant, la liste des noms de quarante-neuf officiers municipaux a été publiée avec beaucoup de fautes dans l'orthographe des noms dans Buchez et Roux, Histoire parlementaire de la Révolution française, op. cit., XXVIII, p. 433. 
nouveau réélu, obtenant 8097 des 10746 suffrages. Cependant, il n'accepta pas son mandat. Au premier tour de l'élection qui a suivi, il y avait 10094 suffrages. Antoine-Marie Bertrand, négociant, obtint 4365 suffrages, tandis que J.E. Gilibert, médecin et modéré, en obtint 3 613. Au second tour, Gilibert obtint 5898 des 11686 suffrages. Cependant, le procureur de la commune, Laussel, le mit en prison par un acte arbitraire et il a démissionné. Le 9 mars 1793 Bertrand était finalement élu ${ }^{45}$.

$\mathrm{Au}$ total, les élections municipales produirent un raz de marée. Cinquante-cinq élus étaient des nouveaux élus ${ }^{4 h}$. La composition sociale, la richesse et la base géographique des conseillers furent bouleversées. Si le maire était négociant, le procureur était un ancien prêtre et son substitut était perruquier. W.D. Edmonds parle même d'une victoire sans précédent des plébéiens sur les propriétaires ${ }^{47}$. Parmi les officiers municipaux, les artisans étaient majoritaires, alors qu'auparavant les hommes d'affaires prédominaient ${ }^{4 x}$. La proportion originaire des sections les plus dépourvues avait doublé ; ils représentaient presque la moitié ${ }^{49}$. Le fait que sept officiers municipaux et sept notables furent ensuite mis en accusation par la Commission populaire montre combien ils étaient radicaux ${ }^{50}$. La victoire électorale des jacobins fut réalisée grâce à ce qu'Edmonds appelle la « démocratie populaire ". Les clubistes ont réussi à obtenir un vote massif en mobilisant les sections pauvres. Les élections avaient été organisées par le Club central, organe des clubs de quartier ${ }^{51}$. Comme les années précédentes, une liste des candidats proposés par ces clubs avait été dressée et distribuée par le Club central. Lalliance des jacobins avec les sectionnaires explique la démocratisation du recrutement social et la radicalisation politique de la municipalité lyonnaise ${ }^{52}$. Cette emprise des radicaux sur le pouvoir municipal a déclenché un mouvement sectionnaire anti-jacobin et antiparisien, renversant la municipalité récemment élue.

(45) Voir Ville de Lyon. Procès-verbaux des corps municipaux de la ville de Lyon, op. cit., III, p. 353391 et IV, p. 1-137.

(46) Le procureur de la commune, son substitut, quinze des officiers municipaux et trente-huit des notables furent des nouveaux élus.

(47) W.D. EDMONDS, Jacobinism and the Revolt of Lyon, op. cit., p. 132.

(48) Ils r'étaient plus que cinq.

(49) W.D. EDMONDs, Jacobinism and the Revolt of Lyon, op. cit., p. 132-133. AD Rhône 1 L 340, Tableau des Ciroyens, Maire, Officiers municipaux, Procureur de la Commune, Substitut du Procureur de la Communc, et Notables composant le Conseil général de la Commune de Lyon, au 5 Décembre 1792, l'an premier de la République française ; BM Lyon Rés 350846, Liste des citoyens éligibles aux places municipales de la ville de Lyon (Lyon, 1790); Paul Frugia, L'Hôtel de Ville de Lyon. L'Hôtel commun et les municipalités lyonnaises, 1789-1795, Lyon, 1985.

(50) Georges Guige, Procès-verbaux des séances de la Commission populaire républicaine et de Salut public de Rhône-et-Loire, 30 juin-8 Octobre 1793, Trévoux, 1899.

(51) $\dot{A}$ Lyon, la section et le quarticr coincident.

(52) W.D.EDMONDs, Jacobinism and the Revolt of Lyon, op. cit., p. 138-143; HANsun, The Jacobin Republic Under Fire, op. cil., p. 142-145; AD Rhône 34 L 1 Registre des procès-verbaux des séances de la Société des Amis de la Constitution, séante à la Croix-Rousse (30 novembre 1792); AD Rhône 31 L 20, Registre des délibérations de la section de Port Saint-Paul ; AD Rhône 31 L 41 Registre des procès-verbaux des assemblées primaires de la section de Bordeaux (Hôtel-Dieu). 
Marseille est le troisième exemple de la démocratisation du recrutement social et de la radicalisation politique qui se sont produites à l'issue des élections au suffrage élargi. Bien que le maire, le procureur et son substitut aient été réélus - mais ils étaient des jacobins ardents - le conseil général fut largement renouvelé : cinquante-trois de ses membres étaient de nouveaux élus ${ }^{33}$. La composition fut renversée, les hommes d'affaires perdant leur domination ${ }^{54}$, les artisans et boutiquiers passant au premier rang; ils représentent au moins $35 \%$ des officiers municipaux et à peu près les trois-quarts des notables ${ }^{55}$. Ce bouleversement s'explique par une participation très forte des artisans et petits producteurs aux assemblées sectionnaires à la fin de l'année 1792, soulignće par Michel Vovelle ${ }^{5 \hbar}$. D'après Malcolm Crook, ce fut le résultat d'une mobilisation dans les quartiers populaires orchestrée par le club jacobin ${ }^{57}$.

En mettant l'accent sur une campagne électorale préalablement organisée par les jacobins, Hanson semble confirmer les thèses de Furet et Gueniffey. Une pétition réclamant l'annulation des élections déclare que nombre de sections ont formé une liste de vingt officiers municipaux et que cette même liste a été proposée à leurs sections respectives ${ }^{5 \%}$. D'après Hanson, ces accusations sont confirmées par les résultats électoraux ${ }^{49}$. Nous proposons une autre conclusion. Le fait que le vote soit presque unanime dans une section ou quelques sections, mais que toutes les sections n'aient pas voté pour les mêmes candidats ne prouve pas qu'il y ait eu concertation dans toutes les sections. Dans la section 13, par exemple, vingt candidats obtinrent 126 suffrages des 127 votants et dix-neuf d'entre eux furent élus officiers municipaux. Dans la section 14, dix-neuf candidats obtinrent 63 à 67

(53) Quatorze des officiers municipaux et trente-neuf des notables furent des nouveaux élus.

(54) Ils ne furent que cinq parmi les officiers municipaux et deux parmi les notables.

(55) La basoche et la médecine ont dispara. Les professions intellectuelles ne sont représentées que par deux maîtres-ès-arts. Aucun noble ou prêtre ne fut élu. AM Marseille $3 \mathrm{~K} 8$ (Procès-verbaux des assemblées primaires); AM Marscille $1 \mathrm{D} 10$ et $1 \mathrm{D} 11$ (Délibérations du conseil municipal) ; AD Bouchesdu-Rhône L 48 (Registre des procès-verbaux des trois corps administratifs de la ville de Marseille). Pour la municipalité de Marseille élue au début de l'année 1790, voir Melvin EDELSTEIN, * Laying the Foundations for the Regeneration of the Empire ", op. cit. 1986, p. $75-95$.

(56) Michel Vovel..E, "Mesure de l'histoire. Le Sans-culotte marseillais ", Histoire et Mesure, I, n" 1,

(57) Malcolm Crook, " Marseille, Aix et Toulon : Vicissitudes du personnel municipal de trois grandes villes provençales à l'époque de la Révolution ", dans Ville et Révolution, sous la direction de Bruno BENOrT, Lyon, 1994, p. 206. Il s'accorde avec mes conclusions sur les artisans et boutiquiers: « on doit attendre les élections du début de 1793 pour saluer leur entrée décisive au-dessus du rang de notable ".

(58) AD Bouches-du-Rhône L 1964. Aux citoyens maire et officiers municipaux, le 11 janvier 1793. Cette pétition était signée par cent cinquante citoyens. Le maire et les officiers municipaux n'ont pas annulé le premier tour des élections dans lesquelles onze des vingt officiers municipaux étaient élus.

(59) Paul Hanson, The Jacobin Republic Under Fire, op. cit., p. 158. Selon lui : " the evidence is very striking. Section 13, for example, submitted a procès-verbal with twenty candidates, each of whom received 126 or 127 votes. All twenty won election, although one resigned and was replaced. In section 14 , too, the same twenty candidates each received between 63 and 68 votes. Other sections were somewhat less blatant, hut a clear pattern emerges. Although not all sections voted the identical slate of candidates, it is obvious that an organized slate had been proposed and that the campaign was ultimately successful $\%$. 
suffrages des 72 votants et dix-huit d'entre eux furent élus. Et dans la section 16, dix-huit candidats obtinrent 94 à 104 des 106 suffrages et dixneuf d'entre eux furent élus. En fait, seules sept des trente-deux sections confirment définitivement les arguments de P. Hanson. En revanche, deux sections (28 et 32 ) infirment ses arguments. Dans la section 32, par exemple, vingt candidats obtinrent 176 à 350 des 363 suffrages, mais dix seulement d'entre eux furent élus. Quant aux élections des notables, seuls huit des trente-deux sections $(16,18,24,25,26,30,31$ et 32$)$ confirment l'interprétation de Hanson. Par contre, quatre sections (1, 27, 28 et 29) infirment ses arguments. Les résultats dans les autres sections ne furent pas aussi nets ${ }^{60}$. La victoire remportée ultérieurement par les sections sur le club montre que le vote fut organisé dans les sections, mais pas forcément par les jacobins ${ }^{\prime \prime}$. Comme à Lyon, la mainmise des jacobins sur la municipalité provoqua une réaction sectionnaire, mais non pas une insurrection. Le 12 juin 1793, la municipalité fut remplacée provisoirement par les commissaires nommés par les sections ${ }^{62}$.

Strasbourg fait figure d'exception. Malgré l'instauration du suffrage élargi, le taux de participation électorale fut plus faible en 1792 qu'en 1791. Ni la composition du conseil général, ni sa modération n'ont été bouleversées. Moins de la moitié des édiles étaient des nouveaux élus ${ }^{6 / 3}$. Le maire était un banquier aisé. Pour à peu près deux-tiers des officiers municipaux, la basoche et les professions intellectuelles dominaient, comme en février 1790. La part des artisans était infime. Elle avait progressé d'un seul officier municipal en 1790 à deux seulement. Si les artisans sont la moitié des notables, ils avaient déjà progressé approximativement à $42 \%$ en $1791^{\text {14. }}$. Le fait que, pour la première fois, les jacobins ont composé une liste de candidats sur laquelle figuraient beaucoup d'artisans et boutiquiers semble conforter les idées de Furet et Gueniffey ${ }^{15 .}$. Mais cette stratégie électorale fut infructueuse malgré un vote faible, et ceci infirme leurs arguments. D'après Hugh Gough, les élections municipales furent une victoire triomphale

(60) Voir AM Marseille 3 K 8.

(61) Voir William ScotT, Terror and Repression in Revolutionary Marseille, New York, 1973, p. 71-126.

(62) AD Bouches-du-Rhône L 1964. Tout d'abord, le maire, Mourraille, et le procureur de la commune, Étienne Seytres, furent acquittés par le Tribunal populaire. Le club fut fermé le 2 juin 1793.

(63) Le substitut du procureur de la commune, cinq des officiers municipaux et quinze des notables étaient des nouveaux élus.

(64) Le procureur de la commune, un avocat, était membre de l'ancien Magistrat. Son substitut, un juriste et publiciste, était administrateur du département. Les artisans et boutiquiers étaient un cinquième des notables en 1790. AM Strasbourg 1 D 3; AM Strasbourg 283 MW 55. Étienne BarTH, Notes biographiques sur les hommes de la Révolution à Strasbourg et les Environs, Mulhouse, 1877.

(65) Je tiens à remercier Claude BLTZINGEER, qui m'a communiqué la liste des candidats présentée le 9 décembre 1792 par la Société des Amis de la Liberté et de l'Égalité, et qui m'a fourni beaucoup de renseignements sur la Révolution à Strasbourg. 
des modérés. Aucun jacobin ne fut élu ${ }^{66}$. Ce succès s'explique par le fait que les sections strasbourgeoises soutinrent les modérés plutôt que les jacobins, mal vus parce qu'ils étaient étrangers ${ }^{67}$. La mainmise des modérés sur la municipalité a été très brève. À l'aide de leurs amis à la Convention, les jacobins se sont débarrassés de cette équipe : le 18 janvier 1793, les commissaires de la Convention l'ont suspendue. Cette fois, les artisans et boutiquiers devinrent dominants ${ }^{\text {txt }}$.

Le cas de Bordeaux ressemble à celui de Strasbourg parce que l'avènement du suffrage élargi n'a pas renversé le personnel municipal et que les sections ont suivi les modérés. Seuls quatre officiers municipaux et la moitié des notables furent des nouveaux élus. Comme Alan Forrest le remarque, la domination du corps municipal par les négociants était même plus marquée qu'en 1790. Le maire, un riche négociant, fut réélu ${ }^{69}$. Les artisans n'avaient guère progressé. Ils étaient toujours quatre parmi les officiers municipaux, mais ils devenaient un peu plus nombreux parmi les notables ${ }^{70}$. À l'opposé de Strasbourg, les jacobins bordelais ont renforcé leur mainmise sur le pouvoir. Même si le maire n'a jamais adhéré, la majorité des édiles

(66) Hugh Gough, "Politics and Power : The Triumph of Jacobinism in Strasbourg, 1791-1793 ", The Historical Joumal, XXIII, 2, 1980, p. 347. Le maire, Turckheim, fils, qui ne fut jamais sociétaire, a très largement battu Euloge Schneider. Si la plupart d'entre eux étaient antérieurement jacobins, vingt-huit ou vingt-neuf des édiles étaient adhérents de la Société dite de l'Auditoire, société scissionnaire du club jacobin.

(67) Voir Hugh Gougin, “Politics and Power ", op. cit., p. 331-336 et p. 351-352.

(68) Je tiens à remercicr Claude BETZinger, qui m'a communiqué la proclamation de la suspension du conseil général de la commune de Strasbourg, le 18 janvier 1793. Seuls trois officiers municipaux et dix-sept notables continuèrent à s'acquitter des fonctions municipales. Voir AD Bas-Rhin 1 L 728 (affiche) et AM Strasbourg 90/874 (Pièces annexes aux délibérations du conseil général de la commune).

(69) D'un peu moins de la moitić, ils passèrent à presque les deux-tiers. AM Bordeaux K 17. Alan Forrest, Society and Politics in Revolutionary Bordeaux, op. cit., p. 122-123. 11 affirme que : " its composition, even more markedly than in 1790 , was dominated by the middle classes and especially by the merchant community. Thirty-one of its members are listed as négociants and a further three as courtiers; but if to these are added two commissionnaires, one commis-négociant, two constructeurs, and two marchands cordiers, it becomes clear that a large majority of the municipal councillors were actively engaged in commerce, mostly in overseas trade. The business ethic was thus very powerful in the ranks of local government, especially as, in addition to these forty-one men, five more were marchands of various descriptions. Of the others, twelve belonged to what might collectively be described as the liberal professions (five lawyers, three doctors, two architects, one chemist, and a teacher). They also included seven artisans or small businessmen : an artist, two carpenters, a clockmaker, a baker, a gunsmith, and a glazier. The council, in short, contained a cross-secton of the powerful Bordeaux bourgeoisic, the men who had gained considerable political power from the Revolution and who were now stubbornly determined to resist encroachments from outside ". Quant à nous, la composition des hommes d'affaires comprend les professions suivantes : le maire (négociant), 11 négociants, 1 marchand et 1 courtier parmi les officiers municipaux et 20 négociants, 2 courtiers, 2 commissionnaires et un marchand parmi les notables. Au total, les hommes d'affaires sont 39 sur 65 édiles, soit $60 \%$. Sont classés comme artisans et boutiquiers, 1 constructeur, 1 pâtissier, 1 vitrier, 1 marchand épicier parmi les officiers municipaux et 2 menuisiers, 2 marchands cordiers, 1 apothicaire, 1 armurier, 1 constructeur, 1 horloger et 1 marchand parfumeur. Au total, les artisans sont 13 sur 65, soit $20 \%$.

(70) Ils ont avancé de six en 1790 et huit en 1791 à neuf. 
étaient clubistes ${ }^{71}$. Cependant, ils avaient déjà accédé au pouvoir en $1791^{72}$. À première vue, Bordeaux paraît conforter les affirmations de Furet et Gueniffey, mais les clubistes bordelais étaient des modérés. Aucun membre du Club national, concurrent plus radical, ne fut élu. Le nouveau conseil municipal soutint la révolte fédéraliste. Dix des édiles firent partie de la Commission populaire. Cinq d'entre eux, y compris le maire, furent mis à mort par la Commission militaire établie par la Convention ${ }^{73}$. Si les élections au suffrage élargi ont abouti à une option modérée, cela s'explique par l'absence de rôle mobilisateur joué par les sections. Comme Lyon, Bordeaux avait un Comité central. Dissout avant les élections municipales, il n'a pas joué le même rôle que le Club central lyonnais. Craignant la manipulation par une minorité, les jacobins et la municipalité s'étaient opposés à la permanence des sections ${ }^{74}$.

Le cas de Rouen ressemble à celui de Bordeaux où le suffrage élargi n'a pas renversé la composition socio-professionnelle du personnel municipal et où les élections quasiment démocratiques ont abouti à l'élection d'une municipalité modérée. Cependant, le changement fut marqué tout particulièrement si on le compare aux élections précédentes. Douze des vingt officiers municipaux étaient de nouveaux élus ; seuls quatre furent reconduits et quatre de plus étaient notables. Le changement fut même plus marqué parmi les notables : trente-six d'entre eux étaient des nouveaux élus, deux seulement étaient réélus et quatre étaient d'anciens officiers municipaux. Si le maire était un nouveau venu, il était ancien administrateur au directoire du département. Le procureur de la commune était l'ancien substitut et le nouveau substitut du procureur était un ancien notable ${ }^{75}$. Même si le maire remplaça un négociant-manufacturier très aisé, il était maître ordinaire à la Chambre des comptes en 1789. Quant au procureur, il était avocat, ayant été élu juge au tribunal du district. Son substitut était un bourgeois, ancien greffier des consuls. Les hommes d'affaires

(71) Le procureur et son substitut furent clublistes. AD Gironde 12 L 19 (Liste des membres de la Société des Amis de la Constitution de Bordeaux, sans date); AD Gironde 12 L 13 (Registre des délibérations de la Sociélé des Amis de la Constitution de Bordeaux).

(72) Alors qu'un tiers des élus cn novembre-décembre 1790 furent jacobins, $58 \%$ des élus en novembre-décembre 179! étaient sociétaires. En janvier 1793, 55-57\% des édiles étaient membres de la Société des Amis de la Liberté et de l'Égalité. Selon Lynn HuNT, "One half of the city councillors of 179091 appear on the only extant membership list of the club. More than one-half $(60 \%)$ of the officials in the "federalist " municipality of 1793 were on the list as compared to only 11 percent of the "terrorist "councillors ". Lynn HunT, Politics, Culture, and Class in the French Revolution, op. cit., p. 201-202.

(73) De plus, un a payé une amende el huit autres furent acquittés. Voir Aurélien VIVIE, Histoire de la Terreur à Bordeaux, 2 vols., Bordeaux, 1877, p. 124,

(74) Sc réunissant à partir de septembre, il fut toutefois fermé par la municipalité le 22 novembre 1792. Voir Alan ForRest, Society and Politics in Revolutionary Bordeaux, op. cit., p. 84-87 et Paul Hanson, The Jacobin Republic Under Fire, op. cit., p. 125-131.

(75) Cependant, selon Félix Cırrembray, seize des officiers municipaux sur vingt, plus le maire et vingt-sept des quarante-deux notables sont de nouveaux venus dans l'assemblée communale. Voir Félix CleRembray, La Terreur à Rouen 1793-1794-1795 D'après des documents inédits, Rouen, 1901, p. 24. 
restent prépondérants Ils formaient la moitié des officiers municipaux et des notables ${ }^{7 h}$. Cependant, leur poids baissa de $80 \%$ des officiers municipaux élus en 1790 et 1791 à $60 \%$, et de $67 \%$ et $75 \%$ des notables nommés auparavant à $52 \%$. Pour la première fois, les artisans et boutiquiers firent leur entrée au corps municipal, mais cette percée fut limitée : seuls quatre étaient élus. Parmi les notables, ils progressèrent d'un en 1791 à cinq"7

Comme à Bordeaux, les jacobins remportèrent la victoire à Rouen. Le maire, le procureur de la commune et son substitut, douze ou treize des vingt officiers municipaux et vingt-huit au moins des quarante-deux notables avaient été adhérents au club jacobin ${ }^{78}$. Cependant, cette mainmise jacobine sur la municipalité ne représentait pas un changement ; les sociétaires détenaient le pouvoir depuis novembre 1791. En fait, la municipalité était divisée entre modérés et futurs montagnards. Selon Félix Clérembray : "Les membres du nouveau conseil général appartenaient effectivement à peu près pour moitié au parti modéré et pour moitié à celui des Montagnards ${ }^{79}$. Comme à Bordeaux, cette modération s'explique par l'absence de rôle mobilisateur joué par les sections. À la veille des élections municipales, le conseil général de la commune rouennaise, dominé par les jacobins, a arrêté que la permanence des sections « est déclarée dissoute ». Il fut défendu aux citoyens de former des assemblées de section, dans le cas où elles ne seraient pas légalement convoquées. Le conseil refusa d'autoriser l'établissement d'un bureau central de réunion de commissaires de sections. De plus, il ordonnait que le président et les secrétaires qui auraient signé la délibération du comité de réunion seraient poursuivis comme fauteurs d'assemblées illicites. Les édiles déclarèrent : «que la permanence des sections n'est pas autorisée par aucune loi, que les circonstances difficiles purent seules solliciter leur convocation; mais que les mêmes motifs n'existant plus, tout doit ployer devant le génie de la loi et de la scrupuleuse observance des principes ${ }^{\star 10}$. Étant donné l'absence de rôle

(76) BM Rouen K 5-6; Ville de Rouen. Analyses des délibérations de l'Assemblée municipale et électorale du 16 juillet 1789 au 4 mars 1790 et du conseil général de la commune du 4 mars 1790 au 25 brumaire an IV, op. cit., p. $89-96$; Vivre en Nomandie sous la Révolution. Documents présentés par Olivier Chaline et Gérard HuRPIN, Tome II, Joumal d'Hocholle, par Gérard HuRPIN, Rouen, 1989, p 457-459. Joumal de Rouen, X, N'. 21, 21 janvier 1793. (Liste des membres composant le conseil général de la commune de Rouen, Année 1793, l'an 2 de la République).

(77) Parmi les officiers municipaux, on compte : un cabaretier, un épicier, un peigneur, et un marchand de vin. Parmi les notables, on compte : un apothicaire, un porteur de toiles, un tavernier, un cafetier et un jardinier. La profession de quatre notables n'est pas déterminée.

(78) Edmond-Jacques CHARDON, Cahiers des procès-verbaux de la Société populaire de Rouen, Rouen, 1909. Je tiens à remercier Danièle Pingué, qui m'a communiqué sa liste des membres de la Société des Amis de la Constitution de Rouen.

(79) Félix Clerembray, La Terreur à Rouen, op. cit., p. 271.

(80) Joumal de Rouen, et du département de la Seine-Inférieure, N". 16716 décembre 1792, p. 909 (Délibération du Conseil général de la Commune de Rouen, en permanence. Séance publique du I3 décembre 1792. l'an premier de la République); Ville de Rouen. Analyses des délibérations de l'Assemblée municipale et électorale du 16 juillet 1789 au 4 mars 1790 et du conseil général de la commune du 4 mars 1790 au 25 brumaire an $I V$, p. 86 . Les élections municipales ont commencé le 23 décembre 1792. 
de coordination joué par le Club central lyonnais, le mouvement sectionnaire rouennais était voué à un échec. Les élections municipales ont donc abouti au succès des négociants, fabricants, et marchands adhérents au club jacobin, comme ce fut le cas à Bordeaux.

Toulouse est également une ville où la démocratisation du recrutement social et la radicalisation politique firent peu de progrès. Alors que le taux de participation électorale montait en flèche, le changement du personnel municipal fut assez restreint. Selon Philippe Nélidoff, les autorités constituées furent dominées encore, dans une large mesure, par les «notables " ${ }^{81}$. Cela est dû au fait qu'il n'y a eu aucun bouleversement du personnel municipal. Un peu plus d'un tiers seulement des édiles étaient des nouveaux élus ${ }^{k_{2}}$. Le maire, le procureur et son substitut étaient toujours des hommes de loi. En revanche, la basoche fut preque éliminée parmi les officiers municipaux ${ }^{83}$. Si les artisans ont progressé légèrement parmi les officiers municipaux, ils ont diminué un peu parmi les notables ${ }^{\text {*4 }}$. L'inverse se produisit pour les négociants ${ }^{\text {s5 }}$. Alors que la municipalité était composée essentiellement de jacobins, Nélidoff affirme qu'elle fut majoritairement girondine $\mathrm{s}^{\mathrm{s}}$. Un peu plus de la moitié des édiles étaient effectivement jacobins, mais les clubistes étaient entrés massivement au conseil général dès l'année précédente ${ }^{k 7}$. Cependant, le maire, le procureur, cinq officiers municipaux et douze notables ont été impliqué ensuite dans le mouvement

(81) Philippe Nfi. IDoff, La Municipalité de Toulouse au début de la Révolution, Toulouse, 1996, p. 216.

(82) Un officier municipal et dix-neuf notables furent des nouveaux élus.

(83) Elle fut représentée pourtant par sept notables. Les négociants, artisans et médecins furent représentés respectivement par quatre officiers municipaux.

(84) Ils avancèrent d'un officier municipal en 1790 à peut-ĉtre quatre, tandis qu'ils tombèrent de treize en 1790 à dix. Nous avons classé parmi les artisans et marchands détaillants un marchand chaussetier et un marchand chapelier. Le premier, antérieurement appelé chaussetier, fut dénommé pourtant négociant en 1792 et l'an II. Il a payé une contribution patriotique de 300 livres, qui signifie un revenu annuel de 1200 livres. Sur la liste des imposables compris dans l'emprunt de six cent millions ordonné par la loi du 19 frimaire an II, Jean-François Caussé est négociant avec une fortune en capital de 40000 livres. Alors que Jean BEYSSi le classe comme artisan, il maintient qu'on peut le regarder comme relativement riche. Voir AM Toulouse 2 G 1-3 (Registres de la contribution patriotique) et 2 G 7 (Relevé des citcyens imposés ou imposables de la Commune de Toulouse devant être compris dans l'emprunt de six cent millions ordonné par la loi du 19 frimaire dernier relativement à leur fortune d'après la notoriété publique) ; Jean BEYsSi, "Le parti jacobin à Toulouse sous te Directoire ", $A H R F$, XXII, 1950, p. 39. Quant à Jean-Joseph Tremoulet, marchand chapelier, il n'a payé que 120 livres de contribution patriotique, ce qui signifie 480 livres de revenu annucl en 1789. Sa fortune en capital était de 20000 livres, la moitié de celle de Caussé.

(85) Ils tombèrent de huit officiers municipaux en 1790 à quatre, mais ils progressèrent de deux notables en 1790 à douze.

(86) Philippe NeI.IDOFf, La Municipalité de Toulouse au début de la Révolution, op. cit., p. 185 et p. 234

(87) AD Haute-Garonne L 4542-4543 (Registre des délibérations de la Société des Amis de la Constitution de Toulouse). 
fédéraliste ${ }^{88}$. En revanche, il y avait une forte minorité de montagnards ${ }^{80}$. Le fait que les résultats électoraux se situent entre ceux de Bordeaux et ceux de Lyon s'explique par le poids moindre des négociants toulousains et notamment le rôle joué par le Comité central des sections. Alors que celui de Bordeaux avait été fermé avant les élections, le comité toulousain ne fut supprimé qu'après l'installation de la municipalité. Il joua un rôle important en étroite collaboration avec le club jacobin. Agissant de concert, les deux essayèrent d'exclure du vote les citoyens entachés d'incivisme. Seuls cinq membres du comité furent toutefois élus notables ${ }^{\text {90 }}$. Il n'est même pas certain que le comité ait dressé et diffusé une liste de candidats aux fonctions municipales ${ }^{21}$. Le comité toulousain fut donc beaucoup plus faible que le club central lyonnais.

En conclusion, Dorvo, de Nantes, avait raison. Pour la première - et unique - fois, les édiles étaient issus "d'une élection purement populaire », selon son expression. Les « éléments populaires », c'est-à-dire les artisans, boutiquiers et marchands détaillants accédèrent plus nombreux au conseil général de la commune. Cependant, notre étude montre qu'il faut nuancer certaines idées reçues. Le corps électoral n'a pas été aussi gonflé que certains historiens le pensent. Les non actifs ne se sont pas toujours mobilisés, Hanson lui-même l'admet, ce fut le cas à Bordeaux et Caen. Alors que le suffrage élargi a fait progresser la démocratisation du recrutement social du personnel municipal, les résultats électoraux infirment certaines affirmations de Hanson. En ce qui concerne le sommet de la municipalité, l'impact de la démocratisation fut limité. La démocratisation de la composition sociale a été plus forte à l'échelon des notables, de moindre importance, qu'au niveau des officiers municipaux. Lavènement du suffrage élargi n'a bouleversé le personnel municipal que dans trois villes ; aucune modification semblable ne s'est produite dans sept autres villes. Cela revient à dire que l'entrée massive des artisans et boutiquiers a eu lieu très souvent en

(88) Le mairc, Marc Derrey, fut guillotiné. Six officiers municipaux et quinze notables étaient suspendus le 13 août 1793 ; l'arrestation de trois notables fut ordonnée le 24 juin 1793, tandis qu'un officier et un notable ont démissionné le 17 mai. Voir la liste dressée par Martyn Lyons, Révolution et Terreur à Toulouse, Toulouse, 1980 , p. 247.

(89) Dix-sept notables et dix officiers municipaux étaient maintenus aux fonctions municipales le 13 août 1793, mais trois d'entre eux ont démissionné le 26 août 1793. Voir Philippe NELIDoff, La Municipalité de Toulouse au début de la Révolution, op. cit., p. 234 et p. 256 n. 110. J. MANDOUL, « Les Municipalités de Toulouse pendant la Révolution ", Recueil de l'Académie de Législation de Toulouse, 1906, $2^{c}$ séric, Tomc II, p. 348-409.

(90) Un orateur déclara qu'ils ont " pour la plupart pourvu aux places dans les diverses administrations ". Le président fut élu le dernier. Léon DuTIL, " Les " communes " en 1792. Le comité central des sections de Toulouse (septembre-décembre) ", Révolution française, LXV, 1913, p. 407.

(91) Voir Philippe Ne1.IDOff, La Municipalité de Toulouse au début de la Révolution, op. cit., p. 194. Selon lui : " Le Comité élabore également une liste de tous les citoyens qui occupent des places publiques et qui sont entachés d'incivisme et une autre des citoyens propres à les remplacer $»$. DUTIL, quant à lui, ne parle pas de telles listes. Voir Léon DuTiL, «Les “ communes" en 1792 ", op. cil., p. 398 et p. $406-407$. 
l'an II grâce aux mesures prises par les représentants en mission, et non par les élections au suffrage élargi. Cela donne raison à Jaurès. Notre étude met en cause les thèses de Furet et Gueniffey. Ils ne se sont pas assez intéressés au contexte politique local de ces élections. Certes, la diffusion des listes de candidats a pris un essor lors de ces élections municipales. Toutefois, à Strasbourg aucun homme figurant sur la liste jacobine n'a été élu. Il faut insister sur le rôle joué par les sections et leur comité ou club central. À Lyon, les élections furent orchestrées par le Club central des sections. C'est la faiblesse du mouvement sectionnaire dans plusieurs villes qui permit aux modérés de l'emporter. Il faut admettre toutefois que ces élections municipales "purement populaires ", ou plutôt au suffrage élargi, marquèrent le progrès de la démocratie pendant la Révolution française.

\section{Melvin EDELSTEIN}

William Paterson University of New Jersey 444 East 82 Street

New York, New York 10028

États-Unis

edelsteinm@wpunj.edu

(Texte français corrigé par Rosine Fry, Ingénieur d'études à l'Université de Bourgogne) 